\title{
HOT-Lines: Tracking Lines in Higher Order Tensor Fields
}

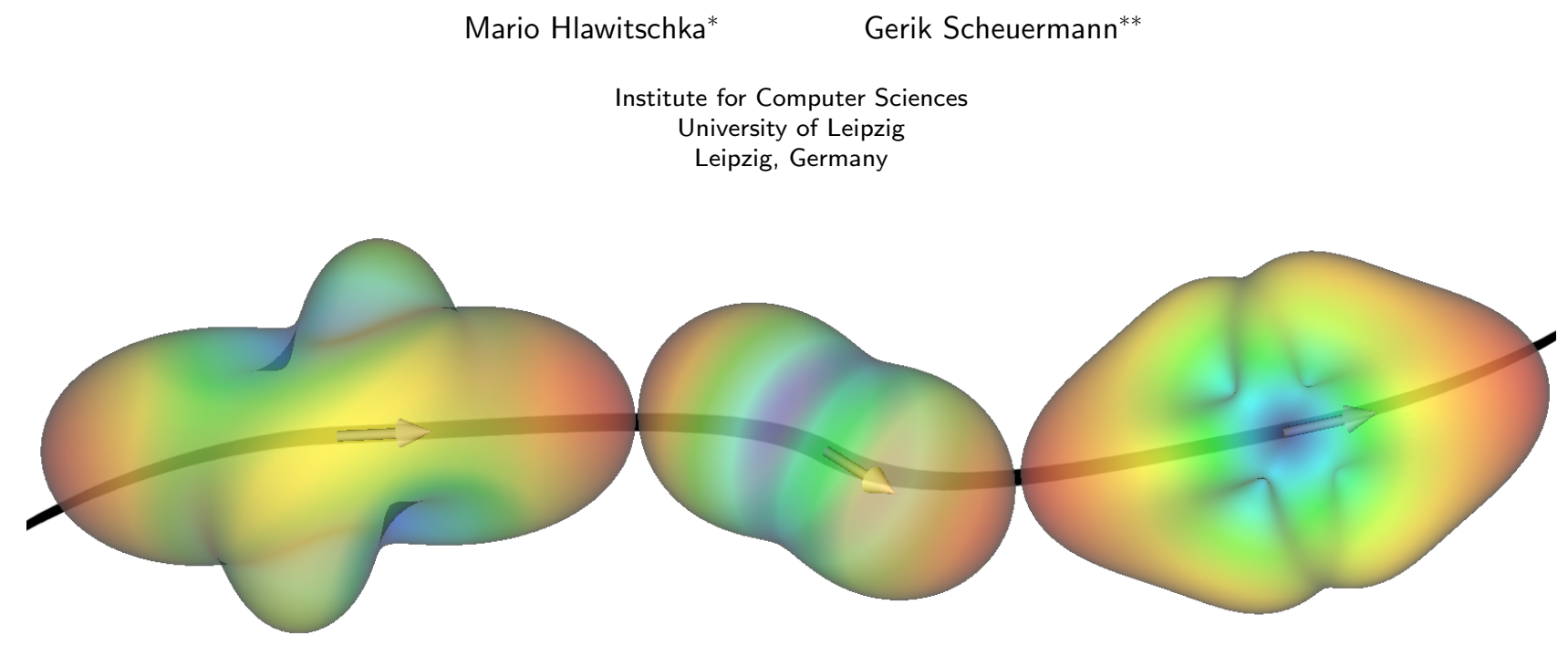

Figure 1: Higher order tensor glyphs and arrows indicating direction of tracking.

\begin{abstract}
Tensors occur in many areas of science and engineering. Especially, they are used to describe charge, mass and energy transport (i.e. electrical conductivity tensor, diffusion tensor, thermal conduction tensor resp.) If the locale transport pattern is complicated, usual second order tensor representation is not sufficient. So far, there are no appropriate visualization methods for this case. We point out similarities of symmetric higher order tensors and spherical harmonics. A spherical harmonic representation is used to improve tensor glyphs. This paper unites the definition of streamlines and tensor lines and generalizes tensor lines to those applications where second order tensors representations fail.

The algorithm is tested on the tractography problem in diffusion tensor magnetic resonance imaging (DT-MRI) and improved for this special application.
\end{abstract}

CR Categories: G.1.2 [Numerical Analysis]: ApproximationSpecial function approximations J.2 [Physical Sciences and Engineering]: Physics- [J.3]: Life and Medical SciencesHealthDiffusion Tensor Imaging

Keywords: Higher order tensors, spherical harmonics, tensor lines, tractography, vector/tensor visualization, visualization in medicine, DT-MRI

\section{INTRODUCTION}

Visual representation of tensors in three dimensional space plays a crucial role in post-processing and data analysis of large-scale finite element model simulation of physical problems. Not only the size of the computed or measured data sets makes it impossible

\footnotetext{
*e-mail: hlawitschka@informatik.uni-leipzig.de

**e-mail: scheuermann@informatik.uni-leipzig.de
}

to visualize the whole data set, but also the complexity in terms of number of values at every data point sometimes makes it impossible to display small subsets of data.

Tensors of fixed order have a long history in scientific computing. Stress and strain tensor in mechanical and civil engineering are described by the well known subset of symmetric second order tensors. Higher tensors are known from material characterization, e.g. the fourth order stiffness tensor $C_{i j k l}$ or the so called "second order stiffness tensor" of order six [10]. Relating visualization, higher order tensors, that are tensors of order greater than two, are unknown.

Another source of tensor data is diffusion tensor magnetic resonance imaging (DT-MRI), where second order tensors are used to represent Gaussian diffusion of water molecules in human brain. Other methods, that will be discussed in detail in section 5, use higher order tensors to model diffusion process along neural fibers.

Many thoughts have been spent on finding optimal glyph icons for tensor fields. A good overview is given by Hashash et al. [9]. Kindlmann [14] used superquadrics to improve visibility of directional components and shape of second order tensors glyphs. Benger et al. [1] proposed the use of semi-transparent glyphs in combination with volume rendering. Also, many ideas of vector field visualization have been adopted to tensor fields, mostly using the eigenvalue-eigenvector decomposition of the matrix representation like tensor lines [3] or adaptions of LIC [33, 23]. Some other methods representing more of the intrinsic properties of the tensor itself have been introduced for volume rendering [15]. To describe the continuous geometric structure of a tensor field, tensor lines $[5,4]$ have been proven to give a good representation of features of the data. They can also be used to display topology graphs of second-order tensor fields [11, 27, 28, 26, 34].

Adaption of most methods to higher order tensor fields fails because the existence of methods similar to the eigenvector decomposition is not known for higher order tensors [18]. Despite of this, directions of largest expansion of the tensor glyph can also be of interest for higher order tensors.

We start by presenting the similarity between a special set of tensors and spherical harmonics and give an example for the use of spherical harmonic representation in tensor visualization. Ex- 
tending previous concepts, we introduce a method of tracking lines in higher order tensor fields, both unifying and generalizing the method of streamlines and tensor lines. Using the spherical harmonic representation, algorithm speed can be improved for symmetric tensor sets of even order.

In section 5, we describe the use of higher order tensors in diffusion tensor imaging. There, detecting neural fibers (a.k.a. the tractography problem) is an important method in understanding connectivity and function of human brain. Because single fibers are far below voxel resolution of the measured data, crossings, kissings and $\mathrm{T}-$ or Y-junctions of fiber bundles present a severe problem to most algorithms. Here, higher order tensor models can give additional information of these structures and can significantly improve tracking in areas of low anisotropy.

\section{SPHERICAL HARMONICS}

The spherical harmonics $Y_{l}^{m}(\theta, \phi)$ are well known as the angular portion of the solution to Laplace's equation in spherical coordinates where azimuthal symmetry is not present [8]. As they represent the eigenfunctions of the Laplace-Beltrami operator, they can be seen as a Fourier transform on the sphere. There are some minor differences between notations in literature. We will use the spherical harmonic basis functions defined by

$$
Y_{l}^{m}(\theta, \phi)=X_{l}^{m}(\theta) e^{\Im m \phi},
$$

where

$$
X_{l}^{m}(\theta)=\left[\frac{2 l+1}{4 \pi} \frac{(l-m) !}{(l+m) !}\right]^{1 / 2} P_{l}^{m}(\cos (\theta))
$$

and

$$
Y_{l}^{-m}(\theta, \phi)=(-1)^{m} Y_{l}^{m *}(\theta, \phi) .
$$

$\mathfrak{I}$ is the complex unit, i.e. $\mathfrak{J}^{2}=-1 . l$ is called the rank or order and $m \in\{-l, \ldots, l\}$ the quantum number. Here, the Condon-Shortley phase $(-1)^{m}$ is already included in the associated Legendre polynomials $P_{l}^{m}$ which are related to the Legendre polynomials

$$
P_{n}(z)=\frac{1}{2 \pi \mathfrak{I}} \oint\left(1-2 t z+t^{2}\right)^{1 / 2} t^{-n-1} d t
$$

by

$$
P_{l}^{m}(x)=(-1)^{m}\left(1-x^{2}\right)^{\frac{m}{2}} \frac{\partial^{m}}{\partial x^{m}} P_{l}(x)
$$

and

$$
P_{l}^{-m}=(-1)^{m} \frac{(l-m) !}{(l+m) !} P_{l}^{m}(x)
$$

Let $\mathbf{g} \in \mathbb{R}^{3}$ be a vector (i.e. $g_{i}$ is a tensor of order one). Then $\mathbf{g}$ can be written in polar coordinates as

$$
\mathbf{g}=g_{i}=\left(\begin{array}{l}
g_{1} \\
g_{2} \\
g_{3}
\end{array}\right)=\left(\begin{array}{c}
r \sin \theta \cos \phi \\
r \sin \theta \sin \phi \\
r \cos \theta
\end{array}\right) .
$$

To shorten notation a bit, we write $\mathbf{g}$ instead of $(\theta, \phi)$ as argument to the spherical harmonic functions and set $r=1$.

Using the scalar product of two functions $f$ and $h$ on the surface of the sphere $S^{2}$ defined by

$$
\langle f, h\rangle_{S^{2}}=\int_{S^{2}} f(\mathbf{g}) h^{*}(\mathbf{g}) d \mathbf{g},
$$

the $Y_{l}^{m}$ provide a fully orthonormal basis in the sense of

$$
\left\langle Y_{l^{\prime}}^{m^{\prime}}, Y_{l}^{m}\right\rangle_{S^{2}}=\delta_{m m^{\prime}} \delta_{l l^{\prime}},
$$

where $\delta_{m n}$ is the Kronecker delta

$$
\delta_{m n}= \begin{cases}1 & \text { iff } m=n \\ 0 & \text { else. }\end{cases}
$$

Any smooth complex function on the sphere can be represented by a Laplace's series

$$
f_{\alpha}(\mathbf{g})=\sum_{l=0}^{\infty} \sum_{m=-l}^{l} a_{l}^{m} Y_{l}^{m}(\mathbf{g}),
$$

where $a_{l m}$ are complex coefficients that can be calculated by the spherical harmonic transformation

$$
a_{l}^{m}=\left\langle f_{\alpha}, Y_{l}^{m}\right\rangle_{S^{2}}
$$

We only focus on real functions, thus $a_{l}^{m}=a_{l}^{-m *}$. This reduces the number of independent coefficients needed for representation. Furthermore $Y_{0}^{0}=\sqrt{(4 \pi)^{-1}}$ is constant, and thus $\sqrt{4 \pi} a_{0}^{0}$ denotes the average value on the sphere. Spherical harmonics of even $l$ are symmetric functions on the sphere and those of odd $l$ are antisymmetric functions. As we only need symmetric functions, all coefficients of odd $l$ are zero. This results in $(l+1)(l+2) / 2$ real coefficients needed to represent those functions of order $l$.

Spherical harmonics of same order $l$ represent rotational invariant subspaces. Another important property of spherical harmonics is that gradients can be calculated analytically.

\section{Higher Order Tensors and SPHERICAL Harmonics}

Let $\mathbf{V}$ be a $d$-dimensional vector space and $\mathbf{V}^{*}$ its dual space. A tensor of type $(r, s)$ is a multilinear form

$$
\sigma=\sigma_{i_{1} \ldots i_{r}}^{j_{1} \ldots j_{s}}: \underbrace{\mathbf{V} \times \ldots \times \mathbf{V}}_{r} \times \underbrace{\mathbf{V}^{*} \times \ldots \times \mathbf{V}^{*}}_{s} \rightarrow \mathbb{R}
$$

We are focusing on $d=3$ and $V=\mathbb{R}^{3}$. When using an orthonormal basis, the dual space $\mathbf{V}^{*}$ and $\mathbf{V}$ can be used interchangeable and for clarity of notation we will write all indices as lower indices.

Let $\hat{g}_{i}$ be a unit vector i.e. $\hat{\mathbf{g}} \|=1$ and let $\sigma_{i_{1}, \ldots, i_{r}}$ be a tensor of rank $r$. We define a scalar function ${ }^{1}$

$$
\begin{gathered}
f_{\sigma}: S^{2} \rightarrow \mathbb{R} \\
f_{\sigma}(\mathbf{g})=\sigma_{i_{1}, \ldots, i_{r}} \cdot \hat{g}_{i_{1}} \cdot \ldots \cdot \hat{g}_{i_{r}}
\end{gathered}
$$

on the unit sphere.

Let $p=\left(p_{1}, \ldots, p_{r}\right)$ be a permutation. A symmetric tensor is a tensor, where

$$
\sigma_{j_{1}, \ldots, j_{r}}=\sigma_{j_{p_{1}}, \ldots, j_{p_{r}}} \quad \forall p .
$$

If $\sigma_{j_{1}, \ldots, j_{r}}$ is symmetric and $r$ even, then $f_{\sigma}$ represents the same set of functions as the truncated Laplace's series of spherical harmonics of order $0 \ldots r$, i.e. for every $\sigma$ there exists a set of coefficients $a_{l}^{m}$ with

$$
f_{\sigma}(\hat{\mathbf{g}})=f_{\alpha}(\hat{\mathbf{g}})=f(\hat{\mathbf{g}})
$$

and vice versa. Formulas for converting both representations analytically can be found in [22].

As spherical harmonics provide an orthogonal basis, tensors of different pure order are orthogonal, too. Amongst others this makes the decomposition of symmetric second order tensors in their trace $\sigma^{S}$, which is the scalar value, and its deviator part (trace free part) $\sigma_{i j}^{D}$ possible.

$$
\sigma_{i j}=\sigma^{S}+\sigma_{i j}^{D}
$$

\footnotetext{
${ }^{1}$ According to Einstein's summing convention we write $a_{i} b_{i}=\sum_{i} a_{i} b_{i}$
} 
which is evaluated as

$$
f_{\sigma_{i j}}(\mathbf{g})=\sigma^{S}+\sigma_{i j}^{D} g_{i} g_{j}
$$

Briefly, every symmetric tensor of arbitrary order can be written as a Laplace's series. This is important, because it shows that higher order approximations of tensor data does not influence the lower order part, and really gives additional information that is not contained in any part of lower order.

In the following sections, we will refer to "tensors of pure order $k$ " when referring to tensors that provide information present in order $k$ only i.e. not present in any other order than $k$.

\subsection{Generalized Tensor Glyphs}

Glyphs are one of the most often used methods in visualization. Single glyphs can be used as interactive probes in the data set or to display additional information on advanced visualization methods. A commonly used tensor glyph for symmetric second order tensors is the ellipsoidal glyph or Cauchy's stress quadric glyph defined by the surface $\mathbf{s}$ with

$$
\sigma_{i j} \mathbf{s}_{i} \mathbf{s}_{j}=\text { const } \text {. }
$$

For diffusion progress this can be interpreted as an iso level of the Gaussian diffusion kernel used in Brownian motion. In anisotropic diffusion, the tensor $\sigma_{i j}$ is proportional to the covariance matrix of the particle movements. Thus the surface describes a level of constant particle density and encloses a volume of higher particle density after a certain diffusion time.

A better model for our purpose is a generalization of Reynold's stress glyph defined by the surface points

$$
\mathbf{s}=\sigma_{i_{1} \ldots i_{r}} \hat{g}_{i_{1}} \cdot \ldots \cdot \hat{g}_{i_{r}} \cdot \hat{g}_{j}
$$

where $\mathbf{g}$ again is a unit vector sampling the sphere (Fig.6). Using $f_{\sigma}$ as defined in Eq.15, this glyph can be drawn for tensors of arbitrary order. Reynold's glyph is quite common in mechanics because of its close relation to Mohr's circle. Different interpretations of this representation in material sciences and geomechanics can be found in a paper by Hashash et al. [9]. Its interpretation for a generalized diffusion is the average distance a particle moves when it is pushed with a certain energy in direction $\hat{\mathbf{g}}$, where the movement has not to be along the direction of the force.

If the tensor values are symmetric and of even order, and many glyphs have to be drawn, the spherical harmonic representation $f_{\alpha}$ from Eq.11 can be used for efficiency. There, the basis functions can be precomputed at the sampling points. A point on the surface is then evaluated by the scalar product of coefficients and basis functions. All points can be evaluated using a single matrix-vector product, where the rows of the matrix contain the pre-evaluated basis functions for the corresponding sampling point. To improve visibility of directional components, maxima of $f$ can be indicated using arrows as shown in the figures 2,3 and 6 .

\section{Higher ORder Tensor Lines}

To define line structures, we need to have a closer look at higher order tensor fields. Let $\Sigma$ be the set of tensors of arbitrary order $r$ and let

$$
\begin{aligned}
& T: \mathbb{R}^{3} \supseteq \quad U \quad \rightarrow \Sigma \\
& \mathbf{p} \rightarrow \sigma(\mathbf{p})
\end{aligned}
$$

be a $C^{2}$ continuous tensor field. In the following, we study the corresponding function $f_{\sigma(\mathbf{p})}$ at each position, i.e.

$$
\begin{aligned}
f_{\sigma(\mathbf{p})}: S^{2} & \rightarrow \mathbb{R} \\
(\theta, \phi) & \rightarrow f_{\sigma(\mathbf{p})}(\theta, \phi)
\end{aligned}
$$
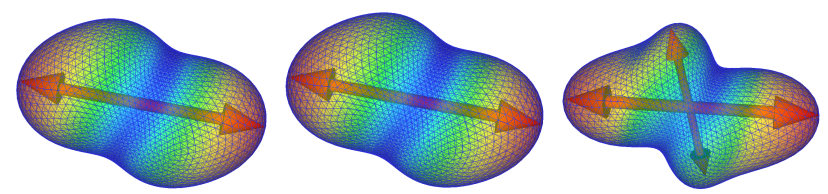

Figure 2: Glyph deforming from single direction to a glyph having two independent directions. Reading from left to right: a new maximum is "born". Tracking horizontal lines goes on without noticing. Reading from right to left: a possible tracking direction vanishes and tracking on the vertical direction stops. Theory shows that there has to be a degenerated tensor between the second and the third.
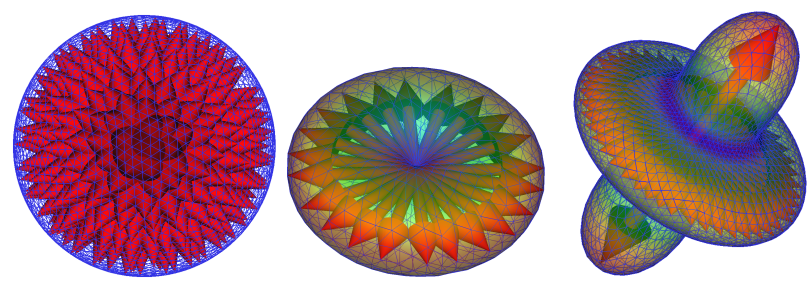

Figure 3: Some degenerated tensors according to Eq.26. Direction ambiguity is shown by displaying some of the possible tracking directions. A completely spherical tensor (left) where $f_{\sigma}$ is constant, a flat tensor (middle) that has a direction ambiguity in a plane. A special case where two maxima are distinct while others are not (right).

so we have a function on the sphere at every position. We call a position $\mathbf{p} \in U$ degenerated if there is a position $(\theta, \phi) \in S^{2}$ where

$$
\nabla_{S^{2}} f_{\sigma(\mathbf{p})}(\theta, \phi)=\mathbf{0}
$$

and

$$
\operatorname{det}\left|\nabla_{S^{2}}^{2} f_{\sigma(\mathbf{p})}(\theta, \phi)\right|=0 .
$$

(The name is well chosen because some tensor lines are not uniquely defined at these positions in accordance to the usual notion of degenerate points introduced by Delmarcelle and Hesselink [3]. Fig. 3 gives a visual impression of some of those tensor glyphs.) Usually testing higher derivatives would lead to a more restrictive definition of degenerated points. There are special instable cases in which study of higher order derivatives would reveal, that what we call degenerated is not degenerated. For simplicity, we ignore these very rare cases in the following sections.

At a regular point (i.e. a not degenerated point) $\mathbf{q} \in U$ we have a finite number $M$ of isolated maxima $\left(\theta_{1}, \phi_{1}\right), \ldots,\left(\theta_{M}, \phi_{M}\right)$ of $f_{\sigma(\mathbf{q})}$. Using the implicit function theorem, we obtain neighborhoods $U_{1}, \ldots, U_{M} \subseteq U$ and unique $C^{1}$-functions

$$
\begin{aligned}
\mathbf{g}_{m}: U_{m} & \rightarrow S^{2} \\
\mathbf{p} & \rightarrow \mathbf{g}_{m}(\mathbf{p})=\left(\theta_{m}(\mathbf{p}), \phi_{m}(\mathbf{p})\right)
\end{aligned}
$$

that parameterize the maxima $\left(\theta_{m}(\mathbf{p}), \phi_{m}(\mathbf{p})\right)$ over the neighborhood $U_{m}$, i.e.

$$
\nabla_{S^{2}} f_{\sigma(\mathbf{p})}\left(\mathbf{g}_{m}(\mathbf{p})\right)=\mathbf{0}
$$

and

$$
\nabla_{S^{2}}^{2} f_{\sigma(\mathbf{p})}\left(\mathbf{g}_{m}(\mathbf{p})\right) \text { is negative definite. }
$$

Using the functions $\mathbf{g}_{m}$, we define unique (major) tensor lines in the tensor field $T$ as curves

$$
\begin{aligned}
x_{m}: I_{m} & \rightarrow U_{m} \\
t & \rightarrow x_{m}(t)
\end{aligned}
$$


with

$$
x_{m}(0)=\mathbf{q}
$$

and

$$
\frac{\partial x_{m}}{\partial t}(t)=\mathbf{g}_{m}\left(x_{m}(t)\right) .
$$

The same proof can be used to define minor tensor lines on $T$ when substituting maxima to minima knowing that

$$
\nabla_{S^{2}}^{2} f_{\sigma(\mathbf{p})}\left(\mathbf{g}_{m}(\mathbf{p})\right)
$$

becomes negative definite. Median higher order tensor lines would be lines following the direction of saddle points of $f_{\sigma}$.

The algorithm for major lines is shown in Algorithm 1 and 2.
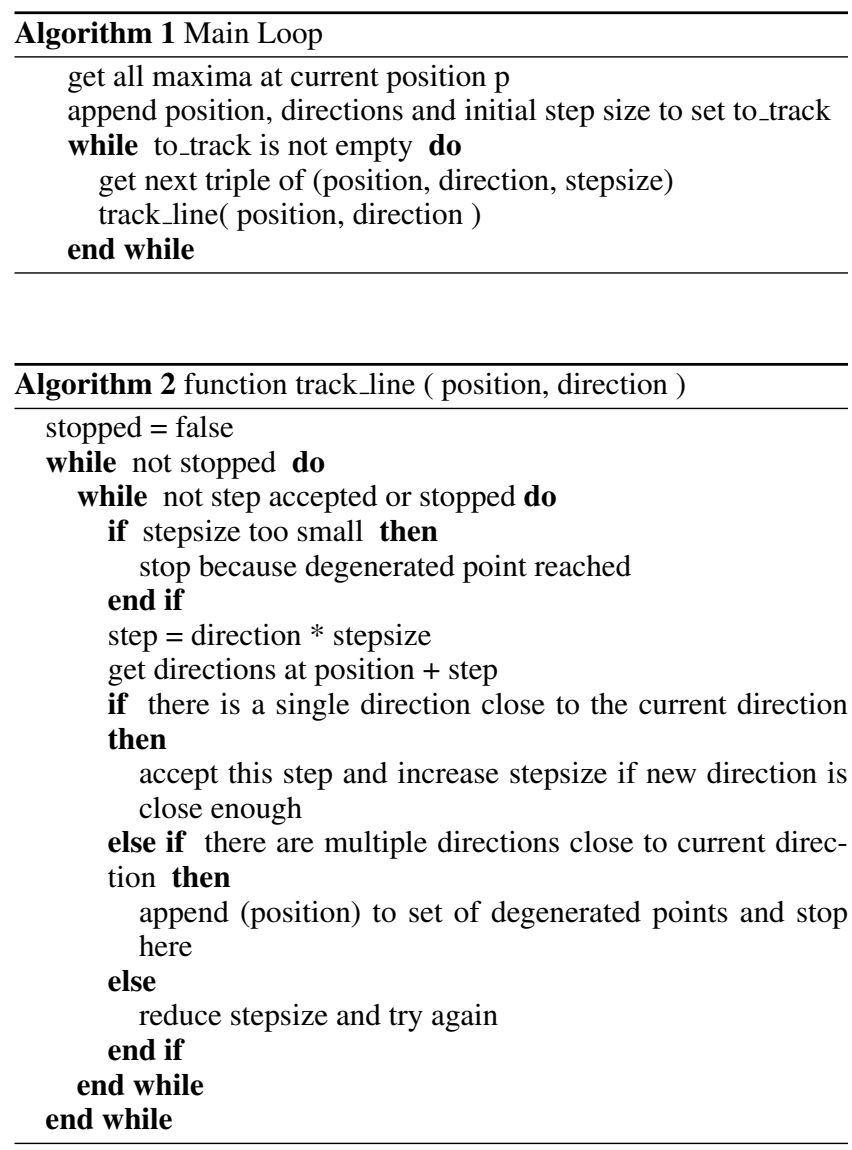

There are only two parameters for our algorithm. One is the stepsize management where lower and upper limits are needed relating to the smoothness of the data set. The second parameter is the direction accuracy which depends on the wanted tracking accuracy and the order of tensors, because higher orders can produce many maxima that are close together.

\subsection{Special Cases}

After having defined a general way of tracking lines in tensor fields, we want to have a look at some of their properties. A degenerated case is the case where tensors are of pure order zero, i.e. they contain only a direction independent scalar component $a_{0}^{0}$, which makes tracking impossible. This is obvious as scalar fields have no orientation so we will ignore scalar fields. Those points can be found as degenerated points or singularities in fields of higher order, too. In second order tensor fields, they are called isotropic and we adopt this nomenclature to fields of arbitrary order. This is a special case of degenerated tensors shown in Fig. 3.

If $\Sigma$ is the set of tensors of order one, i.e. vectors, then it is quite obvious that major higher order tensor lines are equivalent to streamlines, neglecting their parameterization. Minor higher order tensor lines are the same as streamlines on the negated tensor field, i.e. streamlines tracked backwards.

Let $\sigma_{j k}$ be a symmetric tensor of second order and $\lambda_{i}$ its eigenvalues where $\lambda_{i} \geq \lambda_{i+1} \forall i$ and $\mathbf{e}_{i}$ the corresponding eigen directions. Then, if $\sigma_{j k}$ is not degenerated, $f_{\sigma_{j k}}$ has two maxima in direction $\mathbf{e}_{0}$ and $-\mathbf{e}_{0}$. In those areas, HOT-lines are well defined and lead to isolated lines that are equal to second order tensor lines. For major HOT-lines, a degenerated second order tensor has $\lambda_{0}=\lambda_{1}$. The case $\lambda_{0}>\lambda_{1}=\lambda_{2}$ is not degenerated because it does not influence the existence of the two maxima. Medium and minor HOT-lines correspond in a similar way to medium and minor tensor lines.

A physical interpretation for the definition of $f_{\sigma_{j k}}$ can be seen in the following example: Let $\sigma_{j k}$ be a stress tensor (i.e. a symmetric tensor) and $\lambda_{i}$ its eigenvalues and eigenvectors defined as above. The stress on an arbitrary plane with normal $\hat{\mathbf{n}}$ can be computed as

$$
s_{k}=\sigma_{j k} \hat{\mathbf{n}}_{j}
$$

The normal component $\mathbf{n} s$ of $s_{k}$ is

$$
\mathbf{n} s=\sigma_{j k} \hat{\mathbf{n}}_{j} \hat{\mathbf{n}}_{k},
$$

and the vector describing the normal stress is

$$
\mathbf{n} \mathbf{S}=\sigma_{j k} \hat{\mathbf{n}}_{j} \hat{\mathbf{n}}_{k} \hat{\mathbf{n}}_{l}=f_{\sigma_{j k}}(\hat{\mathbf{n}}) \hat{\mathbf{n}} .
$$

This is the same definition as used in the glyph representation in Eq. 21.

Currently, little is known about tensor fields of mixed symmetric and antisymmetric orders. All previously mentioned approaches focus on finding lines in either symmetric second order fields or purely antisymmetric first order tensor fields. Usually, arbitrary second order tensors are decomposed in their trace and its deviator. The deviator is further decomposed into symmetric and antisymmetric part before visualization leading to special interpretations of these components. A good example of this is the second order rate of strain tensor in liquid flow, whose scalar part (trace) denotes the divergence, vector part the rate of rotation in the plane perpendicular to the vector and the symmetric second order part denoting shear flow. Although we are able to track lines in arbitrary tensor fields when using a general description of $f_{\sigma}$ from Eq. 15 , there is currently no physical interpretation of these lines. This will be a topic of further research in tight collaboration with scientists and engineers working in the field of application.

\section{Application in Medical Imaging}

Magnetic resonance imaging (MRI) of water diffusion in tissue has been used to infer anatomical structure and to aid in the diagnosis of many pathologies. This is due to the assumption of diffusion orientation in tissue reflecting the orientation of tissue structures even if these structures are far below the resolution of current imaging techniques. MRI can give useful information about the structure of human brain in vivo and thus helps detecting several diseases like amyotrophic lateral sclerosis [20,24] and the Alzheimer's disease even before other symptoms are visible.

The reconstruction of neural connectivity patterns from DTI is based on the phenomenon of diffusion anisotropy in nerve tissue: Water molecules diffuse relatively freely along the neural fiber direction but are hindered in the fiber-transverse direction. The hindrance of water diffusion in white matter is putatively due to the diffusion barrier presented by the cell membrane and the myelin sheath $[2,16]$. 
Usually, diffusion is represented by a second order tensor model. The tensor anisotropy is related to the amount of fibers and its major eigenvalue to the direction of those fibers. In general, the field can be reduced to an orientationless vector field representing the neural fiber orientations. This model leaves only one important direction per voxel and special features like crossings or bifurcations cannot be represented on voxel scale and are ignored. Thus for voxels containing multiple fibers, other models of diffusion become important $[7,22]$.

\subsection{Theory of DT-MRI}

Diffusional process under influence of magnetic fields in liquids are governed by the Bloch-Torrey differential equation [25, 22]. Solving this equation leads to the Stejskal-Tanner formula for diffusive attenuation

$$
S=S_{0} e^{-\gamma^{2} \sigma^{2} \mathbf{G}(\Delta-\delta / 3) D}=S_{0} e^{-b D},
$$

where $S$ is the signal intensity in presence of a gradient $\mathbf{G}$ and $S_{0}$ is the baseline image which is the signal intensity in the absence of diffusion-sensitizing field gradients to which the remaining measurements can be related. The parameter $b=\gamma^{2} \sigma^{2}(\Delta-\delta / 3)$ is called $b$-factor or diffusion weighting factor. It can be modified by changing the linear field gradient $\mathbf{G}$ of the magnetic field. The gyromagnetic ratio $\gamma$ of a nucleus relates the Larmor frequency $\omega_{0}$ in a static magnetic field $\mathbf{B}$ to its strength by $\omega_{0}=\gamma|\mathbf{B}|$. Results of the measurement strongly depend on the chosen $b$-values as pointed out by Frank [6] and Jones [12].

The scalar value $D$ defining the strength of signal attenuation is called apparent diffusion coefficient in the direction of measurement and is the value measured by MRI. In diffusion tensor imaging, directional information is important and a symmetric second order tensor $D_{i j}=D_{j i}$ is reconstructed from at least six independent gradient weighted directions in addition to the $S_{0}$ image. It is obvious that more complex functions on the sphere can be used to approximate the angular dependency of the measured diffusion coefficient. Because the measurement can only evaluate motion along gradients, but not its direction, all reconstructed data representing particle movements has to be symmetric on the sphere. We reconstruct higher order symmetric second order tensors $D_{i_{0} \ldots i_{r}}$ of order $r$ by solving Eq. 40 by least square fit.

\subsection{Tensor Construction}

Frank [6] described the use of the spherical harmonic transform as a method of deriving the spherical harmonic coefficients. In general this is not a trivial task. In analogy to the fast Fourier transform, there exist fast spherical harmonic transformations but they need special sets of sampling points which may not be available in the MRI scanner hardware and software.

Thus, we use least square fitting of gradient magnitudes to the spherical harmonic basis as this is a widely used method for second order tensors and makes comparison of our results easier. There are better methods when outliers are present that have been presented by Mangin et al. [17]. These can be used, too, but we did not notice such problems on our data sets.

For fitting the data, the gradient directions have to be chosen thoughtfully because they provide a fundamental part of the matrix describing the relation between the diffusion tensor coefficients and the measured apparent diffusion coefficients. If it is not well conditioned, the reconstruction may be instable and the tensor values overshoot. It seems like a common method to circumvent this problem by using a higher amount of gradient directions (about 80 up to more than 120) which increases the time of measurement or forces to reduce image resolution which both is clinically not reasonable.

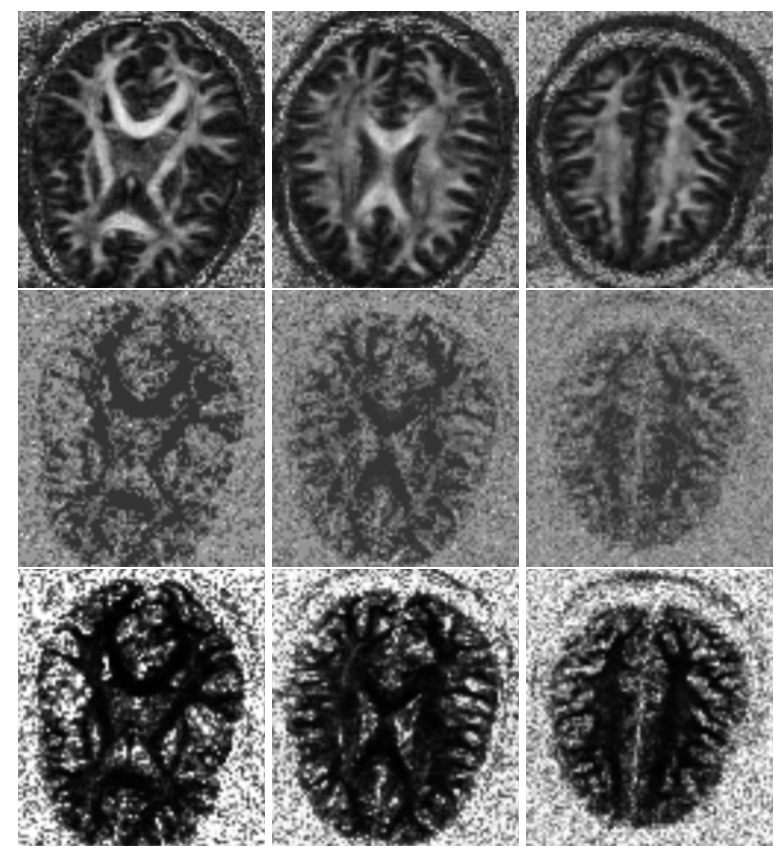

Figure 4: Comparison between fractional anisotropy (top), number of tracking directions (middle) and multi fiber index (bottom) of the same slices of an MRI scan. Diffusion tensors have order 4. On all images, white means high values, black is zero. For the direction map, black means zero or two directions, white ten or more. The FMI is clipped to a range of $[0 \ldots 2]$ for higher contrast of the images.

As the time of measurement is a limiting factor in medical applications, one has to find the best trade-off between measuring time and number of gradient directions which are directly related to the highest possible order in tensor representation. Special analysis of this topic has been done by Jones et al. [13]. We use two different sets of gradient directions for our experiments. A standard set of 36 directions provided by the scanner and the set of 30 gradient directions described by Jones which enable us to reconstruct symmetric tensors of order four and order six respectively.

Because of the properties of diffusion respectively the method of measurement, all reconstructed tensors are symmetric and consist only of even order information. The property of positive definite second order tensors can be found in $f_{\sigma}>0$. This means that the coefficient $a_{0}^{0}$ in spherical harmonic representation must be large enough to compensate the sum of oscillations of any higher spherical harmonic. Usually, in measured data this may not be true because of noise, but neither did we experience such problems in the valid part of our data sets (i.e. inside the measured head) nor has the component $a_{0}^{0}$ an influence on the stability of our approach.

Whereas the medical visualization community agrees on the fact that the assumption of Gaussian diffusion for reconstruction of second order tensors gives good results for simple, single-fiber structures, more work has to be done for higher order tensor data. There, methods like Q-Space imaging and Q-Ball imaging [31,30] have been developed and used to compute the orientation distribution function (ODF), a smooth function on the sphere describing the probability of a particle to move in a certain direction on the sphere. As we target the clinical usability of fiber tracking, Q-Space imaging is not usable because of higher data acquisition times. Different methods have been proposed to compute fiber directions using the ODF. Because of the relatively small angular resolution of our clinical data sets, there is only little difference in the results and we use the maximum of the ODF as a fast approximation. 


\subsection{Notes on Anisotropy Measurements}

In medicine and computer science there exist many different methods to calculate measurements for the anisotropy of tensor values. Many of them, like fractional anisotropy

$$
\mathrm{FA}=\sqrt{\frac{3}{2} \frac{\sum_{i}\left(\lambda_{i}-\bar{\lambda}\right)^{2}}{\sum_{i} \lambda_{i}^{2}}}, \quad \bar{\lambda}=\frac{1}{3} \sum_{i} \lambda_{i}
$$

or linear and planar anisotropy used e.g. by Westin et al. [32] are based only on eigenvalues $\lambda_{i}$ of the second order tensors. Because of the described orthogonality of tensors, they do not reveal any information about higher order components. Other methods have been proposed like the standard deviation or the variance of diffusion coefficients along the gradient directions or other sampled grids [6]. A relatively good method is calculating the relation of energy in higher order spherical harmonics to those of second order, labeled fractional multi fiber index (FMI) by Frank [7]

$$
\mathrm{FMI}=\frac{\sum_{l>2} \sum_{m}\left|a_{l}^{m}\right|^{2}}{\sum_{m}\left|a_{2}^{m}\right|^{2}} .
$$

There are instabilities, when both tensor components of order two and of higher orders are close to zero. Thus, we propose to introduce a threshold on the overall energy for calculation. Fig. 4 compares three different anisotropy measures. The first row displays the fractional anisotropy, that only depends on the eigenvalues of the second order tensor. The second row shows the number of lines present in each voxel, i.e. the number of local maxima of the function $f$ described earlier. It can be seen that the multi fiber index shown in the third row is clearly related to the number of directions found in the voxel. In addition, both, the number of fibers per voxels and the multi fiber index are inverse to the fractional anisotropy in highly oriented areas. It is worth to note that direction ambiguity increases in areas where fiber bundles get thinner especially when reaching the cortex.

\subsection{Fiber Crossings and T-Junctions}

The basic idea of using higher order representations of the diffusion function is to preserve more information of the measured data for its analysis. All current approaches in diffusion tensor tractography have similar problems at highly isotropic regions of low fractional anisotropy where major and middle eigenvalue have approximately the same value and the tensor glyph approximates a donut shape (Fig. 3b). These areas can be divided into mainly isotropic areas (spherical shape, Fig. 3a) and those really having two main directions, i.e. two crossing fiber bundles, that cannot be represented by simple second order tensor models.

Higher order models reveal more information about the local structure, because they do not reduce information to only six values where only five indicate directional information out of about thirty values measured in commonly used DTI scanners. All other information is ignored in the preprocessing step before knowing whether local structure allows this reduction or whether further analysis needs this information. Thus crossings can be detected and multiple directions can be computed, representing the fiber directions. From the definition of higher order tensor lines, it can be easily seen that those areas having fiber crossings must be surrounded by a surface of degenerated tensors.

The problem of T-junctions is mentioned in literature from time to time. While it is not clear if single fibers have T-junctions or not, fiber bundles may have. Despite this fact, the measured diffusivity at those positions will never have $\mathrm{T}$ or $\mathrm{Y}$ shapes because of the symmetry enforced by the measurement and the resolution of voxels. This does not lead to wrong results, but reflects the nature of diffusion.
Some of the problems mentioned may be solved by using additional information about the local neighborhood like smoothing data along lines, smoothing in areas of similar behavior [19, 29] or preserving directions when possible [21]. Those algorithms can not reconstruct local behavior inside the voxel and thus use less information than possible. Obviously, they can be combined with our approach to improve the quality and give more information about the global structure.

\subsection{Speed Improvements for Fiber Tracking}

When tracking fibers in human brain, usually many thousand paths are precalculated and reduced afterwards to fiber bundles. Thus, tracking single fibers has to be a fast operation. To improve speed, we fall back to simple second order tensor fiber tracking algorithms in areas where only one dominant fiber direction is present. Therefore, our fiber tracking algorithm monitors local anisotropy measurements and orientation change to decide whether to go on or analyze higher order tensor components. These approximations can be made due to the similarity of HOT-lines with second order tensor lines presented in section 4.1 , and the fact that anisotropy values give a good hint of the number of directions present in DT-MRI data sets as shown in Fig. 4. The modified fiber tracking algorithm is shown in algorithm 3 .

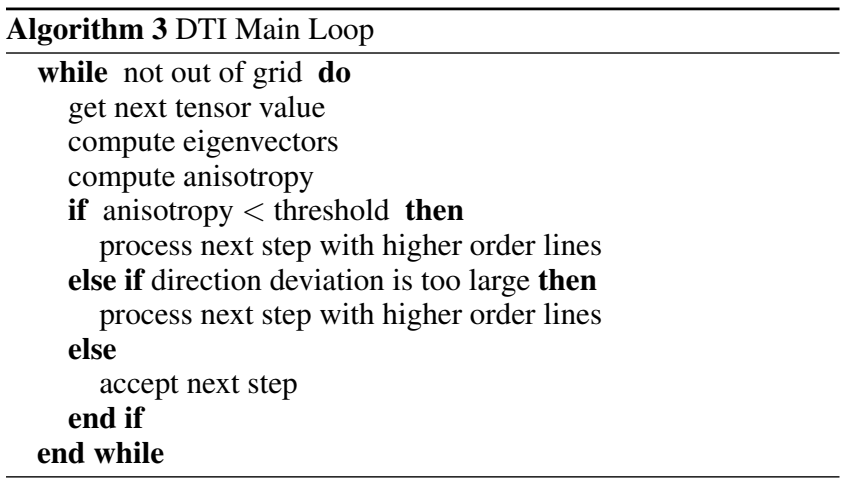

There anisotropy may be any anisotropy measurement indication the influence of multiple directions in a voxel. We can further modify algorithm 1 to track lines through degenerated parts if there exist isolated maxima by using algorithm 4 .

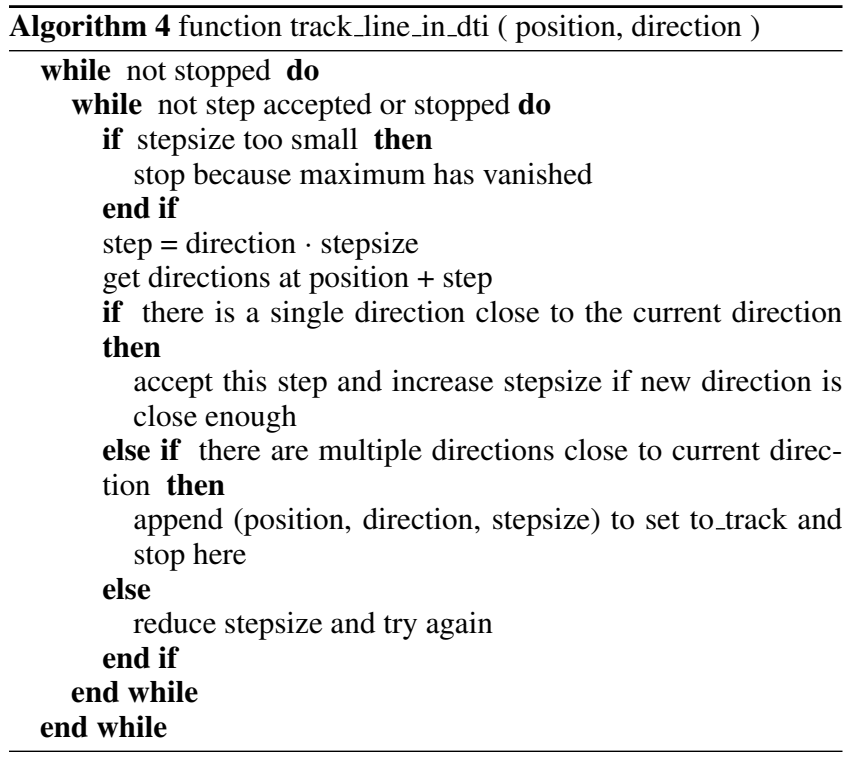




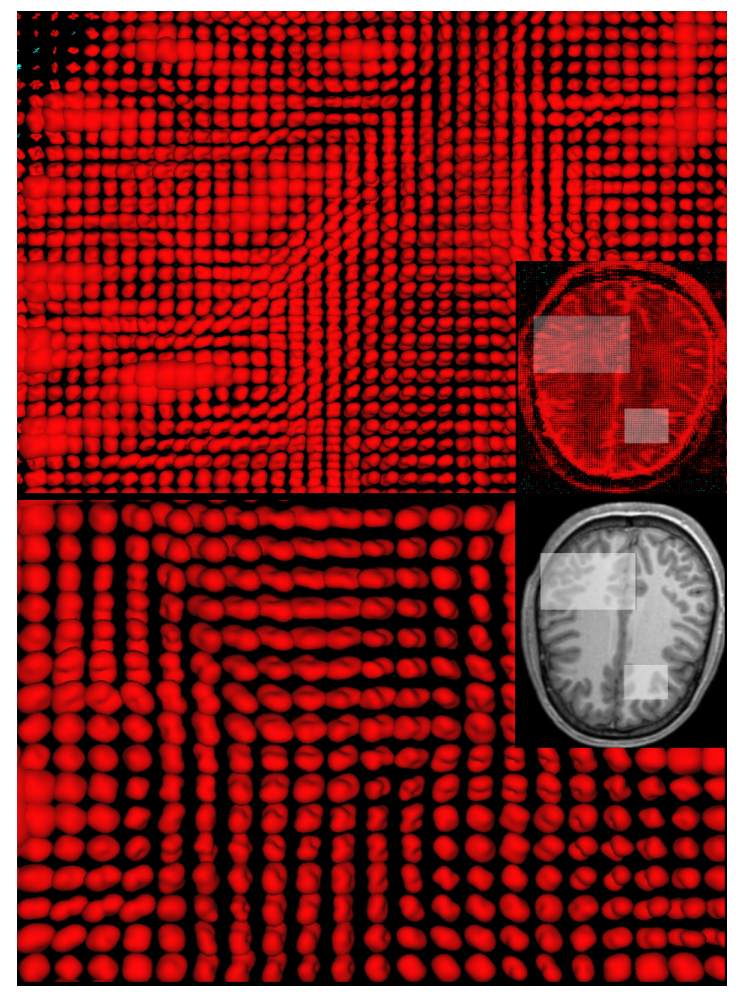

Figure 5: Fourth order tensor glyphs painted at a slice of DT-MRI data. The smaller images are the complete slice of diffusion tensor glyphs (top) and the T1 weighted image (bottom) provided for orientation. The two highlighted areas in these images indicate the areas displayed in the top and bottom image resp.

Usually thresholds of fractional anisotropy are chosen between 0.2 and 0.3 , depending on the signal to noise ratio (SNR) of the data set. Even though the algorithm does not explicitly depend on a special representation of the tensor values, we recommend the spherical harmonic representation of Eq. 11 because this enables better speed optimization of the algorithm.

\subsection{Data Acquisition}

Whole-head high-resolution DT images were acquired from a healthy subject between the ages of 20-30 years. The diffusion tensor was computed as described previously from diffusion weighted SE-EPI (TR $8100 \mathrm{~ms}$; TE $120 \mathrm{~ms} ; 1.7 \times 1.7 \times 3 \mathrm{~mm}^{3} ; 2$ acq.) with $b$-factors 0 and $1000 \mathrm{~s} / \mathrm{mm}^{2}$ (36 directions). The resulting data consists of $128 \times 88 \times 33$ voxel. Because of restrictions due to the chosen gradient directions, only tensors of order four could be calculated which leads to additional noise reduction.

\subsection{Implementation}

All presented methods have been implemented in $\mathrm{C}++$ using the FAnToM visualization system developed at the University of Leipzig. First, existing methods [21] have been implemented to be able to compare our results. Integration itself has been done using Euler's method with adaptive stepsize control, second order RungeKutta and dopri5/RK45 working on the tensor data set to get correct interpolation of eigenvectors and to be able to prevent flipping of eigenvector directions in regions of high isotropy. Maxima were found by sampling the function $f$ on a triangulated grid. The steps of tracking lines in highly isotropic areas of the MRI data set can be seen in Fig. 7.

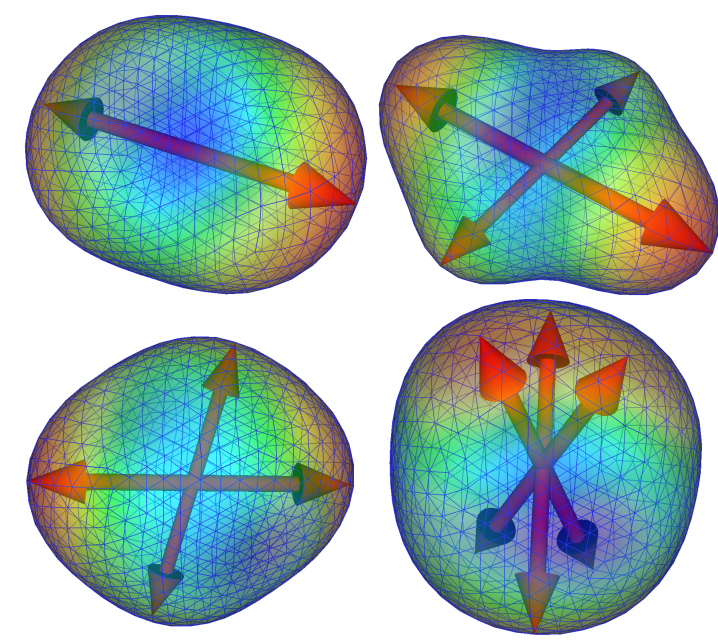

Figure 6: Different glyphs of symmetric tensors of order four taken from the MRI data set. The glyph surface is sampled on a triangulated grid created by subdivision of an icosahedron. Possible tracking directions are indicated by arrows.

\section{CONClusion ANd Future Work}

We have developed a framework for visualizing higher order tensor fields by presenting a fast method for displaying glyphs for symmetric tensors of even order. The theoretical concept of higher order tensor lines has been introduced. Their existence has been proved as well as their relation to second order tensor lines and streamlines. Furthermore, the concept is generalizes to all types of data specifying smooth scalar functions in spherical coordinates at every point of the data set.

Application to diffusion tensor imaging has proved to be useful on scanned data sets, where fiber crossings and low signal to noise ratios make it impossible to follow fiber tracts using the information provided by a simple Gaussian second order tensor diffusion model. We have shown that even with a relatively small number of directions it is possible to use higher order tensor models for diffusion which makes it possible to use these models in clinical application. Future work needs to be done on investigating the signal to noise ratio in relation to increased $b$-values to find a optimal tradeoff between higher order structures revealed by higher $b$-values and SNR. Furthermore, the definition of better anisotropy values is a crucial point for speed improvements of our algorithm on diffusion tensor data. Here, information about local signal to noise ratio may be introduced, too.

Having the well founded definition of HOT-lines, further applications have to be investigated which will be done in close cooperation with scientists and engineers of the respective field of application especially mechanics, where possible applications could be displaying properties of complex composite materials like metalmatrix composites.

\section{ACKNOWLEDGEMENTS}

First of all, we want to thank all members of the FAnToM development team for providing the framework for our implementation. Special thanks go to Alfred Anwander, Marc Tittgemeyer, Thomas R. Knösche and Harald E. Möller from the Max Planck Institute of Cognitive NeuroScience Leipzig for the fruitful discussion and for providing the data sets. 


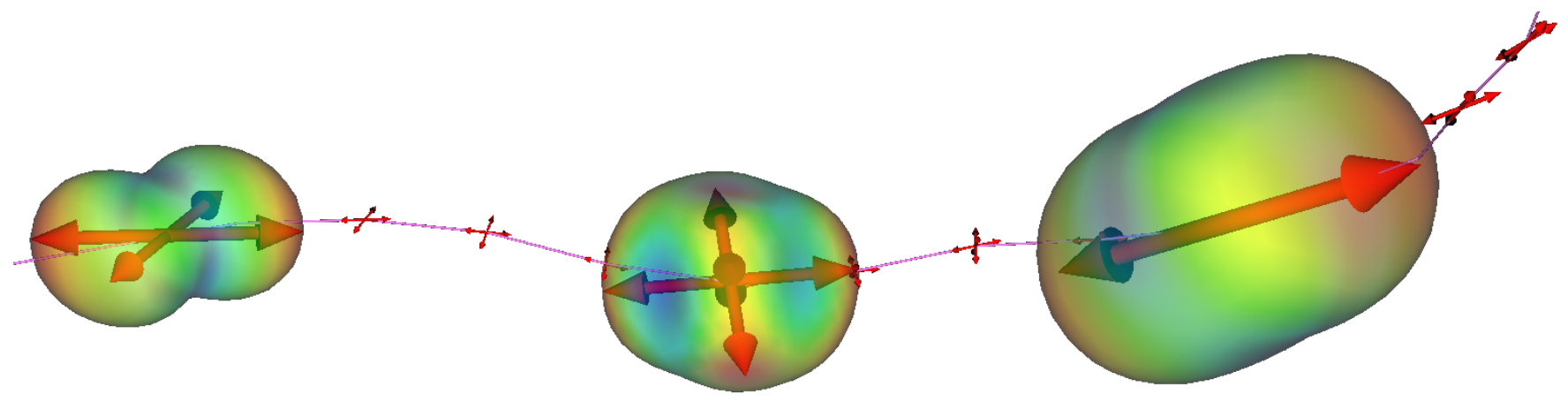

Figure 7: Results from the tracking algorithm on spherical harmonics of order four in the MRI data set. Tracking proceeds from left to right. The implementation continues tracking on degenerated points (i.e. points where the number of possible tracking direction changes.)

\section{REFERENCES}

[1] W. Benger and H.-C. Hege. Tensor splats. In Proceedings of the Conference on Visualization and Data Analysis, 2004.

[2] D. L. Bihan and P. van Zijl. Editorial: From the diffusion coefficient to the diffusion tensor. NMR in Biomedicine, 2002.

[3] T. Delmarcelle and L. Hesselink. Visualization of second order tensor fields and matrix data. In Proceedings of IEEE Visualization 1992, page 316. IEEE, CS Press, 1992.

[4] T. Delmarcelle and L. Hesselink. Visualizing second-order tensor fields with hyperstreamlines. IEEE Computer Graphics and Application, 13 (4)(4):25-33, July/August 1993.

[5] R. R. Dickenson. A unified approach to the design of visualization software for the analysis of field problems. In SPIE Proceedings, volume 1083, Bellinham, Washington, 1989. SPIE - The International Society for Optical Engineering.

[6] L. R. Frank. Anisotorpy in high angular resolution diffusion-weighted MRI. Magnetic Resonance in Medicine, pages 935-939, 2001.

[7] L. R. Frank. Characterization of anisotropy in high angular resolution diffusion-weighted MRI. Magnetic Resonance in Medicine, pages 1083-1099, 2002.

[8] W. Freeden, T. Gervens, and M. Schreiner. Constructive Approximation on the Sphere (With Applications to Geomathematics). Oxford Sciences Publication, Clarendon Press, Oxford, 1998.

[9] Y. M. A. Hashash, J. I.-C. Yao, and D. C. Wotring. Glyph and hyperstreamline representation of stress and strain tensors and material constructive response. International Journal for Numerical and Analytical Methods in Geomechaics, 27:603-626, 2003.

[10] K. Helbig. A formalism for the consistent description of non-linear elasticity of anisotropic media. Institut franćais du pétrole, 1998.

[11] L. Hesselink, Y. Levy, and Y. Lavin. The topology of symmetric, second-order 3D tensor fields. IEEE Transactions on Visualization and Computer Graphics, 3(1):1-11, 1997.

[12] D. K. Jones and P. J. Basser. Squashing peanuts and smashing pumpkins: How noise distorts diffusion-weighted MR data. Magnetic Resonance in Medicine, 2004

[13] D. K. Jones, M. Horsfield, and A. Simmons. Optimal strategies for measuring diffusion in anisotropic systems by magnetic resonance imaging. Magnetic Resonance in Medicine, pages 515-525, 1999.

[14] G. Kindlmann. Superquadric tensor glyph. Joint EUROGRAPHICS IEEE TCVG Symposium on Visualization, 2004.

[15] G. L. Kindlmann, D. M. Weinstein, and D. Hart. Strategies for direct volume rendering of diffusion tensor fields. IEEE Transactions on Visualization and Computer Graphics, 6 (2):124-137, 2000.

[16] D. Le Bihan. Looking into the functional architecture of the brain. Neuroscience, 4, June 2003

[17] J.-F. Mangin, C. Poupon, C. Clark, D. L. Bihan, and I. Bloch. Eddycurrent distortion correction and robust tensor estimation for MR diffusion imaging. Elseview Science, 2002.

[18] C. D. M. Martin. Tensor decompositions workshop discussion mynotes. American institude of Mathemattics (AIM), July 2004.
[19] T. McGraw, B. Vemuri, Y. Chen, M. Rao, and T. Mareci. DT-MRI denoising and neuronal fiber tracking. Medical Image Analysis, 8, 2004.

[20] D. Miller, R. Grossman, S. Reingold, and H. McFarland. The role of magnetic resonance techniques in understanding and managing multiple sclerosis. Brain, pages 3-24, Jan 1998.

[21] S. Mori and P. C. M. van Zijl. Fiber tracking: principles and strategies - a technical review. NMR in Biomedicine, 2002.

[22] E. Özerslan and T. H. Mareci. Generalized diffusion tensor imaging and analytical relationships between diffusion tensor imaging and high angular resolution diffusion imaging. Magnetic Resonance in Medicine, pages 955-965, 2003.

[23] A. Siegfridsson, T. Ebbers, E. Heiberg, and L. Wigström. Tensor field visualization using adaptive filtering of noise fields combined with glyph rendering. In Proceedings of IEEE Visualization '02, pages 461-469, Los Alamitos, CA, 2001. IEEE Computer Society Press.

[24] A. T. Toosy, D. J. Werring, R. W. Orell, R. S. Howard, M. D. King, G. J. Baker, D. H. Miller, and A. J. Thompson. Diffusion tensor imaging detecs corticospinal tract involvement at multiple levels in amyotrophic lateral sclerosis. Neurol Neurosurgy Psychiatry, 74:1250$1257,2003$.

[25] H. C. Torrey. Bloch equations with diffusion terms. Physical Review, 104(3):563-565, November 1956

[26] X. Tricoche. Vector and Tensor Field Topology Simplification, Tracking, and Visualization. $\mathrm{PhD}$ thesis, University of Kaiserslautern, Germany, 2002.

[27] X. Tricoche, G. Scheuermann, and H. Hagen. A topology simplification method for $2 \mathrm{D}$ vector fields. In D. Ebert, M. Gross, and B. Hamann, editors, Proceedings of IEEE Visualization 2000, pages 359-366, Los Alamitos, CA, 2000. IEEE Computer Society Press.

[28] X. Tricoche, G. Scheuermann, and H. Hagen. Tensor topology tracking: A visualization method for time-dependent 2D symmetric tensor fields. In Eurographics 2001 Proceedings, Computer Graphics Forum 20(3), pages 461-470, Saarbrücken, Germany, 2001. The Eurographics Association.

[29] D. Tschumperle and R. Deriche. Diffusion tensor regularization with constraints preservation. In Computer Vision and Pattern Recognition 2001, Kauai, Hawaii, 2001.

[30] D. S. Tuch. Q-ball imaging. Magnetic Resonance in Medicine, pages 1358-1372, 2004.

[31] D. S. Tuch. Diffusion MRI of Complex Tissue Structure. PhD thesis, Massachusetts Institute of Technology, 2002.

[32] C.-F. Westin, S. E. Maier, B. Khidhir, P. Everett, F. A. Jolesz, and R. Kikinis. Image processing for diffusion tensor magnetic resonance imaging. In Proceedings of Second Int. Conf. on Medical Image Computing and Computer-assisted interventions, pages 441-452, 1999.

[33] X. Zheng and A. Pang. Hyperlic. In Proceedings of IEEE Visualization '03, pages 249-256, Los Alamitos, CA, 2003. IEEE Computer Society.

[34] X. Zheng and A. Pang. Topological lines in 3D tensor fields. Proceedings of IEEE Visualization 2004, 2004. 